\title{
MIXED FINITE ELEMENT METHODS FOR FRACTIONAL NAVIER-STOKES EQUATIONS*
}

\author{
Xiaocui $\mathrm{Li}^{1)}$ \\ College of Mathematics and Physics, Beijing University of Chemical Technology, \\ Beijing 100029, China \\ Email: anny9702@126.com,xiaocuili@mail.buct.edu.cn \\ $\mathrm{Xu} \mathrm{You}$ \\ Department of Mathematics and Physics, Beijing Institute of Petrochemical Technology, \\ Beijing 10261\%, China \\ Email: youxu@bipt.edu.cn
}

\begin{abstract}
This paper gives the detailed numerical analysis of mixed finite element method for fractional Navier-Stokes equations. The proposed method is based on the mixed finite element method in space and a finite difference scheme in time. The stability analyses of semi-discretization scheme and fully discrete scheme are discussed in detail. Furthermore, We give the convergence analysis for both semidiscrete and fully discrete schemes and then prove that the numerical solution converges the exact one with order $O\left(h^{2}+k\right)$, where $h$ and $k$ respectively denote the space step size and the time step size. Finally, numerical examples are presented to demonstrate the effectiveness of our numerical methods.
\end{abstract}

Mathematics subject classification: 60N15, 65M60, 60N30, 75D05.

Key words: Time-fractional Navier-Stokes equations, Finite element method, Error estimates; Strong convergence.

\section{Introduction}

The purpose of the present paper is to study the error estimates of the mixed finite element method for the incompressible fractional Navier-Stokes equations

$$
\begin{cases}u_{t}+\mathscr{B}^{\alpha} \mathscr{L} u+u \cdot \nabla u+\nabla p=f, & \text { in } \Omega \times[0, T], \\ \nabla \cdot u=0, & \text { in } \Omega \times[0, T], \\ u(x, 0)=u_{0}, & \text { in } \Omega, \\ u=0, & \text { on } \partial \Omega \times[0, T],\end{cases}
$$

where $\Omega \subset \mathbf{R}^{2}$ is a bounded and connected polygonal domain, $u$ represents the velocity field, $p$ is the associated pressure, $u_{0}$ is the initial velocity and $f$ is an external force, $\mathscr{L} u=-\nu \Delta u$ ( $\nu>0$ is the viscosity coefficient), $\mathscr{B}^{\alpha}:={ }^{R} D_{t}^{1-\alpha}$ is the Riemann-Liouville fractional derivative in time defined by: for $0<\alpha<1$,

$$
\mathscr{B}^{\alpha} \varphi(t):=\frac{\partial}{\partial t} \mathscr{I}^{\alpha} \varphi(t):=\frac{\partial}{\partial t} \int_{0}^{t} \omega_{\alpha}(t-s) \varphi(s) d s \text { with } \omega_{\alpha}(t):=\frac{t^{\alpha-1}}{\Gamma(\alpha)}
$$

\footnotetext{
* Received July 18, 2018 / Revised version received July 30, 2018 / Accepted November 28, 2019 / Published online January 17, 2020 /

1) Corresponding author
} 
with $\mathscr{I}^{\alpha}$ being the temporal Riemann-Liouville fractional integral operator of order $\alpha$.

The above-mentioned problem has many physical applications in many areas such as heterogeneous flows and materials, turbulence, viscoelasticity and electromagnetic theory. Particularly when $\alpha=1$, the problem (1.1) reduces to the classical Navier-Stokes equations, numerical approximations of which have been studied by many authors $[1-4,8-19,25,32,34,37-44,46,47]$. However, for the fractional Navier-Stokes equations (FNSE) which are nonlinear in character, most of them do not have exact analytical solutions. It is shown that very few cases in which the exact solution of fractional Navier-Stokes equations can be obtained, where it have to make certain assumptions about the state of the fluid and a simple configuration for the flow pattern is to be considered. Hence it is necessary to analyze and study the approximation and numerical techniques of FNSE. However, to our best knowledge, numerical analysis of such problem for fractional Navier-Stokes equations is missing except $[27,56]$ in the literature. Therefore, this article aims to fill the gap, study and obtain the strong convergence approximations of FNSE like (1.1).

In recent years, there have been numerous studies on fractional diffusion equation. Lin and $\mathrm{Xu}[31]$ have proposed the finite difference scheme in time and Legendre spectral methods in space for the time-fractional diffusion equation. Deng [6] has established the stability and error estimates for the time fractional Fokker-Planck equation and then proved that the convergent order is $O\left(k^{2 \alpha}+h^{\mu}\right)$, where $k$ is the time step size and $h$ is the space step size. Liu et al. [35] have developed a two-grid algorithm based on the mixed finite element method for a nonlinear fourthorder reaction-diffusion equation with the time-fractional derivative of Caputo-type. Jin et al. [21], by using piecewise linear functions, have studied two semidiscrete approximation schemes, i.e., Galerkin finite element method and lumped mass Galerkin finite element method, for the homogeneous time-fractional diffusion equation. Zeng et al. [51] have studied the second-order accurate schemes for the time-fractional diffusion equation with unconditional stability based on finite element method in space and the fractional linear multistep methods in time. Besides, some other interesting works in this aspect can be found in $[5,7,20,22-24,30,33,36,45,52-55,57]$.

In this article, our goal is to give some detailed numerical analysis of the mixed finite element method for the problem (1.1). On one hand, the discretization in space is done by the mixed finite element method. First of all, the velocity is split into two parts by introducing a linearized discrete problem with solution $v_{h}$. In particular, Motivated by the Ritz-Volterra projection, we then introduce the fractional Stokes-Volterra projection $S_{h} u$, the role of which is similar to that of a Ritz projection in treating the heat equation. Subsequently, with virtue of the property of the operator $E_{h}$ as well as the standard duality arguments, the $L^{2}$-error estimate for the velocity is shown. On the other hand, firstly following the idea of Zhuang et al. [53] that has discretized the Riemann-Liouville fractional derivative $\mathscr{B}^{\alpha}$ in time, then we adopt the finite difference method and obtain the stability and convergence properties related to the time discretization. The stability analyses of semi-discretization scheme and fully discrete scheme are discussed in detail. Furthermore, We give the convergence analysis for both semidiscrete and fully discrete schemes and prove that the numerical solution converges the exact one with order $O\left(h^{2}+k\right)$, where $h$ and $k$ respectively the space step size and the time step size.

The structure of this paper is as follows: In section 2, we introduce some preliminaries and notations, give the definition of the Mittag-Leffler function. In Section 3, we introduce the notations for finite element spatial semidiscretization, describe the semidiscrete Galerkin approximations about space and establish the error estimate for the velocity. In Section 4, we present several lemmas which play a crucial role in the proof of the error estimate of the 
time discretization. We firstly give the fully discrete scheme for (1.1) and then obtain the error estimate for the fully discrete scheme. Finally, in Section 5, numerical examples are presented to demonstrate the effectiveness of our numerical methods.

\section{Preliminaries}

Throughout the paper, we denote as $C$ a constant that may not be of the same form from one occurrence to another, even in the same line. In this section, we introduce some notations and recall the definition of Mittag-Leffler function .

We use the standard notation $H^{s}(\Omega),\|\cdot\|_{s},(\cdot, \cdot)_{s}, s \geq 0$ for the Sobolev spaces, the standard Sobolev norm and inner product, respectively. When $s=0, L^{2}(\Omega)$ is written instead of $H^{0}(\Omega)$, the $L_{2}$-inner product and $L_{2}$-norm are separately denoted by $(\cdot, \cdot)$ and $\|\cdot\|$. For the mathematical setting of problem (1.1), the following spaces

$$
X=H_{0}^{1}(\Omega)^{2}, \quad Y=L^{2}(\Omega)^{2}, \quad M=L_{0}^{2}(\Omega)=\left\{q \in L^{2}(\Omega): \int_{\Omega} q d x=0\right\},
$$

are introduced. Next, let the closed subset $V$ of $X$ be given by

$$
V=\{v \in X, \quad \operatorname{div} v=0\}
$$

and denote by $H$ the closed subset of $Y$, i.e.,

$$
H=\left\{v \in Y, \quad \operatorname{div} v=0,\left.v \cdot n\right|_{\partial \Omega}=0\right\} .
$$

Moreover, the continuous bilinear form $d(\cdot, \cdot)$ on $X \times M$ and the bilinear operator $B(\cdot, \cdot)$ on $X \times X$ are separately defined by

$$
\begin{array}{ll}
d(\phi, p)=(\operatorname{div} \phi, p), & \forall \phi \in X, \quad \forall p \in M, \\
B(u, v)=(u \cdot \nabla) v+\frac{1}{2}(\operatorname{div} u) v, & \forall u, v \in X .
\end{array}
$$

At the same time, a trilinear form $b(\cdot, \cdot, \cdot)$ on $X \times X \times X$ is introduced by

$$
b(u, v, w)=(u \cdot \nabla v, w), \quad u, v, w \in X,
$$

which has the following properties (cf., [13] [10]):

$$
\begin{array}{ll}
b(u, v, w)=-b(u, w, v), \quad b(u, v, v)=0, & \forall u, v, w \in X, \\
\|b(u, v, w)\| \leq M\|\nabla u\|\|\nabla v\|\|\nabla w\|, & \forall u, v, w \in X .
\end{array}
$$

For the readers convenience, we give the definition of Mittag-Leffler function. We shall use extensively the Mittag-Leffler function $E_{\alpha, \beta}(z)[26]$ defined by

$$
E_{\alpha, \beta}(z)=\sum_{k=0}^{\infty} \frac{z^{k}}{\Gamma(k \alpha+\beta)}, z \in \mathbb{C},
$$

where $\Gamma($.$) is the standard Gamma function defined as$

$$
\Gamma(z)=\int_{0}^{\infty} t^{z-1} e^{-t} \mathrm{~d} t, \mathfrak{R}(z)>0 .
$$




\section{Space Semi-Discretization}

In this section, we will give the variational formulation of (1.1), describe the semidiscrete Galerkin approximations and then derive the error estimates for the velocity about space discretization. From now on, we denote by $h$ with $0<h<1$ a real positive discretization parameter tending to zero.

\subsection{Semidiscrete fractional Navier-Stokes equations}

Let $A(\cdot, \cdot): X \times X \rightarrow \mathbb{R}$ be the bilinear form associated with the operator $\mathscr{L}=-\nu \triangle$ which is symmetric and positive definite on $X$. With the notations in Section 2, the variational formulation of (1.1) is as follows: find $(u, p) \in(X, M)$ for all $t \in[0, T]$ such that for all $(\phi, q) \in(X, M)$

$$
\left\{\begin{array}{l}
\left(u_{t}, \phi\right)+A\left(\mathscr{B}^{\alpha} u, \phi\right)+b(u, u, \phi)-d(\phi, p)+d(u, q)=(f, \phi), \\
u(x, 0)=u_{0}
\end{array}\right.
$$

We introduce the finite element subspace $\left(X_{h}, M_{h}\right)$ of $(X, M), Y_{h} \subset Y$ and define the subspace $V_{h}$ of $X_{h}$ given by

$$
V_{h}=\left\{v_{h} \in X_{h}, \quad \operatorname{div} v_{h}=0\right\} .
$$

We assume that the couple $\left(X_{h}, M_{h}\right)$ satisfies the discrete LBB(or named inf-sup) condition

$$
\sup _{v_{h} \in X_{h}} \frac{\left(\varphi_{h}, \nabla \cdot v_{h}\right)}{\left\|\nabla v_{h}\right\|} \geq \beta\left\|\varphi_{h}\right\|, \quad \forall \varphi_{h} \in M_{h}
$$

where $\beta>0$ is a constant.

Let $P_{h}: Y \rightarrow V_{h}$ denotes the $L^{2}$-orthogonal projection defined by

$$
\left(P_{h} v, v_{h}\right)=\left(v, v_{h}\right), \quad v \in Y, \quad v_{h} \in V_{h} .
$$

For the space $M_{h}$ we assume that, for each $q \in H^{1}(\Omega) \cap M$, there exists an approximation $j_{h} q \in M_{h}$ such that

$$
\left\|q-j_{h} q\right\| \leq \operatorname{ch}\|q\|_{1},
$$

where $c$ is a positive constant, independent of $h$.

The operator $E_{h}(t)$ is introduced by

$$
E_{h}(t) v_{h}=\sum_{j=1}^{\infty} E_{\alpha, 1}\left(-\lambda_{j}^{h} t^{\alpha}\right)\left(v, \varphi_{j}^{h}\right) \varphi_{j}^{h}, \quad v_{h} \in X_{h},
$$

where $\left\{\lambda_{j}^{h}\right\}_{j=1}^{N}$ and $\left\{\varphi_{j}^{h}\right\}_{j=1}^{N}$ are respectively the eigenvalues and the eigenfunctions of the discrete Laplace operator $\mathscr{F}:=-\nu \Delta_{h}$ defined by

$$
-\left(\nu \Delta_{h} \psi, \chi\right)=\nu(\nabla \psi, \nabla \chi), \quad \forall \psi, \chi \in X_{h} .
$$

For later use, we need the regularity result which is related to the operator $E_{h}(t)$ and collect the result in the next lemma. 
Lemma $3.1([\mathbf{2 2}])$. Let $E_{h}(t)$ be defined by (3.3) and $\psi \in X_{h}$. Then it holds that

$$
\left\|E_{h}(t) \psi\right\|_{p} \leq c t^{\frac{-\alpha(p-q)}{2}}\|\psi\|_{q},
$$

where $\alpha \in(0,1), q \leq p$ and $0 \leq p-q \leq 2$.

The discrete analogue of weak formulation (3.1) now reads as follows: find $\left(u_{h}, p_{h}\right) \in$ $\left(X_{h}, M_{h}\right)$ such that for all $\left(\phi_{h}, q_{h}\right) \in\left(X_{h}, M_{h}\right)$,

$$
\left(u_{h t}, \phi_{h}\right)+A\left(\mathscr{B}^{\alpha} u_{h}, \phi_{h}\right)+b\left(u_{h}, u_{h}, \phi_{h}\right)-d\left(\phi_{h}, p_{h}\right)+d\left(u_{h}, q_{h}\right)=\left(f, \phi_{h}\right),
$$

with $u_{h}(0)=P_{h} u_{0}$.

For the discrete approximation, it is straightforward to verify that the trilinear term $b\left(u_{h}, v_{h}\right.$, $w_{h}$ ) enjoys the following properties (cf., [14]):

$$
\begin{array}{ll}
b\left(u_{h}, v_{h}, w_{h}\right)=-b\left(u_{h}, w_{h}, v_{h}\right), \quad b\left(u_{h}, v_{h}, v_{h}\right)=0, & \forall u_{h}, v_{h}, w_{h} \in X_{h}, \\
\left\|b\left(u_{h}, v_{h}, w_{h}\right)\right\| \leq M\left\|\nabla u_{h}\right\|\left\|\nabla v_{h}\right\|\left\|\nabla w_{h}\right\|, & \forall u_{h}, v_{h}, w_{h} \in X_{h} .
\end{array}
$$

\subsection{Error estimate for the velocity}

With $V_{h}$ as above, we now introduce an equivalent Galerkin formulation. Find $u_{h} \in V_{h}$ such that $u_{h}(0)=P_{h} u_{0}$ and for $t>0$

$$
\left(u_{h t}, \phi_{h}\right)+A\left(\mathscr{B}^{\alpha} u_{h}, \phi_{h}\right)+b\left(u_{h}, u_{h}, \phi_{h}\right)=\left(f, \phi_{h}\right), \quad \forall \phi_{h} \in V_{h} .
$$

For $f \in L^{2}(\Omega)$, problem (3.8) is uniquely solvable for all $t>0$. Indeed, setting $\phi_{h}=u_{h}$ in (3.8) and recalling the property $b\left(u_{h}, u_{h}, u_{h}\right)=0$, we obtain

$$
\frac{1}{2} \frac{d}{d t}\left\|u_{h}\right\|^{2}+A\left(\mathscr{B}^{\alpha} u_{h}, u_{h}\right)=\left(f, u_{h}\right) .
$$

On integration with respect to the temporal variable $t$ and using the following property $\mathscr{B}^{\alpha}$ has satisfied $([28])$

$$
\int_{0}^{t} A\left(\mathscr{B}^{\alpha} u_{h}, u_{h}\right) d s \geq c_{\alpha} T^{\alpha-1} \int_{0}^{t}\left\|\nabla u_{h}\right\|^{2} d s
$$

we observe that

$$
\left\|u_{h}(t)\right\| \leq\left\|u_{h}(0)\right\|+t\|f\| .
$$

Thus, by classical theorems of the theory of ordinary differential equations, (3.8) has a unique global solution $u_{h}(t), t>0$.

The next theorem summarizes the error bound about space discretization.

Theorem 3.1 (Error estimate for space discretization). Let $u$ and $u_{h}$ be the solutions of (3.1) and (3.8), respectively, then the following estimate holds

$$
\left\|u-u_{h}\right\| \leq C h^{2},
$$

where $C=c\left(\|u\|_{2}+\mathscr{I}^{1-\alpha}\|p\|_{1}+\left\|u_{t}\right\|_{2}+\mathscr{I}^{1-\alpha}\left\|p_{t}\right\|_{1}\right)$. 
We firstly dissociate the non-linearity by introducing an intermediate solution $v_{h}$. Let $v_{h}$ be a finite element Galerkin approximation to a linearised time-fractional Navier-Stokes equation satisfying

$$
\left(v_{h t}, \phi_{h}\right)+A\left(\mathscr{B}^{\alpha} v_{h}, \phi_{h}\right)+b\left(u, u, \phi_{h}\right)=\left(f, \phi_{h}\right), \quad \forall \phi_{h} \in V_{h},
$$

with $v_{h}(0)=P_{h} u_{0}$. Subsequently, the error is split as

$$
e:=u-u_{h}=\left(u-v_{h}\right)+\left(v_{h}-u_{h}\right)=: \xi+\eta \text {. }
$$

Note that $\xi$ is the error committed by approximating a linearized (Stokes) problem and $\eta$ represents the error due to the presence of the nonlinearity in the equation.

Define the fractional Stokes-Volterra projection $S_{h} u \in V_{h}$ such that

$$
A\left(\mathscr{B}^{\alpha}\left(u-S_{h} u\right), \phi_{h}\right)=\left(p, \nabla \cdot \phi_{h}\right), \quad \phi_{h} \in V_{h},
$$

that is,

$$
A\left(\left(u-S_{h} u\right), \phi_{h}\right)=\mathscr{I}^{1-\alpha}\left(p, \nabla \cdot \phi_{h}\right), \quad \phi_{h} \in V_{h},
$$

where $\mathscr{I}^{1-\alpha}$ denotes the temporal fractional integral operator with singular kernel $t^{-\beta} / \Gamma(1-\beta)$. The form of the above projection is motivated by the Ritz-Volterra projection, see Lin [29] for parabolic integro-differential equations and by the elliptic projection introduced by Wheeler [49] for parabolic initial and boundary value problems.

Below, We now decompose $\xi$ as

$$
\xi:=\left(u-S_{h} u\right)+\left(S_{h} u-v_{h}\right)=: \zeta+\theta .
$$

First of all, we derive the error bound for $u-S_{h} u$.

Lemma 3.2. For the fractional Stokes-Volterra projection $S_{h} u$, we have

$$
\begin{aligned}
& \left\|u-S_{h} u\right\|+h\left\|\nabla\left(u-S_{h} u\right)\right\| \leq c h^{2}\left(\|u\|_{2}+\mathscr{I}^{1-\alpha}\|p\|_{1}\right), \\
& \left\|\left(u-S_{h} u\right)_{t}\right\|+h\left\|\nabla\left(u-S_{h} u\right)_{t}\right\| \leq c h^{2}\left(\left\|u_{t}\right\|_{2}+\mathscr{I}^{1-\alpha}\left\|p_{t}\right\|_{1}\right) .
\end{aligned}
$$

Proof. Setting $\phi_{h}=P_{h} u-S_{h} u$ in (3.13) yields

$$
\begin{aligned}
& \nu\left\|\nabla\left(u-S_{h} u\right)\right\|^{2}=A\left(u-S_{h} u, u-S_{h} u\right) \\
= & A\left(u-S_{h} u, u-P_{h} u\right)+\mathscr{I}^{1-\alpha}\left(p-j_{h} p, \nabla \cdot\left(P_{h} u-S_{h} u\right)\right),
\end{aligned}
$$

and hence

$$
\left\|\nabla\left(u-S_{h} u\right)\right\| \leq \operatorname{ch}\left(\|u\|_{2}+\mathscr{I}^{1-\alpha}\|p\|_{1}\right) .
$$

Now we introduce the following dual problem,

$$
-\Delta w+\nabla z=u-S_{h} u
$$

then it satisfies the following regularity result

$$
\|w\|_{2}+\|z\|_{1} \leq c\left\|u-S_{h} u\right\|
$$


Hence we obtain

$$
\begin{aligned}
& \left\|u-S_{h} u\right\|^{2}=\left(-\Delta w, u-S_{h} u\right)+\left(\nabla z, u-S_{h} u\right) \\
= & \frac{1}{\nu} A\left(u-S_{h} u, w\right)-\left(z, \nabla \cdot\left(u-S_{h} u\right)\right) \\
= & \frac{1}{\nu} A\left(u-S_{h} u, w-P_{h} w\right)+\frac{1}{\nu} \mathscr{I}^{1-\alpha}\left(p-j_{h} p, \nabla \cdot P_{h} w\right)-\left(z, \nabla \cdot\left(u-S_{h} u\right)\right) \\
= & \frac{1}{\nu} A\left(u-S_{h} u, w-P_{h} w\right)+\frac{1}{\nu} \mathscr{I}^{1-\alpha}\left(p-j_{h} p, \nabla \cdot P_{h} w\right)-\left(z-j_{h} z, \nabla \cdot\left(u-S_{h} u\right)\right) \\
\leq & c h\left\|\nabla\left(u-S_{h} u\right)\right\|\left(\|w\|_{2}+\|z\|_{1}\right)+c h^{2} \mathscr{I}^{1-\alpha}\|p\|_{1}\|w\|_{2} \\
\leq & c h\left\|\nabla\left(u-S_{h} u\right)\right\|\left\|u-S_{h} u\right\|+\operatorname{ch}^{2} \mathscr{I}^{1-\alpha}\|p\|_{1}\left\|u-S_{h} u\right\| .
\end{aligned}
$$

This clearly implies (3.14). The second inequality (3.15) is proved similarly.

Now we are in a position to estimate $\xi$. Since $\xi=\zeta+\theta$ and the estimates of $\zeta$ are known from the Lemma 3.2, it is sufficient to estimate $\theta$.

Lemma 3.3. For $\xi=u-v_{h}$, it satisfies the following estimate

$$
\|\xi\| \leq c h^{2}\left(\|u\|_{2}+\mathscr{I}^{1-\alpha}\|p\|_{1}+\left\|u_{t}\right\|_{2}+\mathscr{I}^{1-\alpha}\left\|p_{t}\right\|_{1}\right) .
$$

Proof. Subtracting (3.12) from (3.1), the equation in $\xi$ is written as

$$
\left(\xi_{t}, \phi_{h}\right)+A\left(\mathscr{B}^{\alpha} \xi, \phi_{h}\right)=\left(p, \nabla \cdot \phi_{h}\right), \quad \phi_{h} \in V_{h} .
$$

To complete the estimate for $\xi$, we only need to estimate $\theta$. The equation in $\theta$ reads as

$$
\left(\zeta_{t}, \phi_{h}\right)+\left(\theta_{t}, \phi_{h}\right)+A\left(\mathscr{B}^{\alpha} \zeta, \phi_{h}\right)+A\left(\mathscr{B}^{\alpha} \theta, \phi_{h}\right)=\left(p, \nabla \cdot \phi_{h}\right), \quad \forall \phi_{h} \in V_{h} .
$$

Making use of the definition of $S_{h} u$, then the above equation can be simplified as

$$
\left(\theta_{t}, \phi_{h}\right)+A\left(\mathscr{B}^{\alpha} \theta, \phi_{h}\right)=-\left(\zeta_{t}, \phi_{h}\right) .
$$

Let $\phi_{h}=\theta$ in the above equation, there holds

$$
\frac{1}{2} \frac{d}{d t}\|\theta\|^{2}+A\left(\mathscr{B}^{\alpha} \theta, \theta\right)=-\left(\zeta_{t}, \theta\right)
$$

On integration with respect to the temporal variable $t$, we obtain that

$$
\|\theta\|^{2}+\int_{0}^{t} A\left(\mathscr{B}^{\alpha} \theta, \theta\right) d s \leq\|\theta(0)\|^{2}+\int_{0}^{t}\left\|\zeta_{t}\right\|\|\theta\| d s .
$$

With (3.10), we derive

$$
\|\theta\| \leq\|\theta(0)\|+\int_{0}^{t}\left\|\zeta_{t}\right\| d s .
$$

By virtue of Lemma 3.2, we obtain

$$
\|\theta\| \leq \operatorname{ch}^{2}\left(\left\|u_{t}\right\|_{2}+\mathscr{I}^{1-\alpha}\left\|p_{t}\right\|_{1}\right)
$$


By the triangle inequality, we have

$$
\|\xi\| \leq\|\zeta\|+\|\theta\| \leq c h^{2}\left(\|u\|_{2}+\mathscr{I}^{1-\alpha}\|p\|_{1}+\left\|u_{t}\right\|_{2}+\mathscr{I}^{1-\alpha}\left\|p_{t}\right\|_{1}\right),
$$

which completes the proof.

Subsequently, we give the main result in this section. By making use of the properties of the operator $E_{h}$ and the standard duality arguments, the methods of which are different from classical Navier-Stokes equations, we derive the error estimate for the velocity.

Proof of Theorem 3.1. Since $e=u-u_{h}=\left(u-v_{h}\right)+\left(v_{h}-u_{h}\right)=\xi+\eta$ and the estimate of $\xi$ is known from Lemma 3.3, it is enough to estimate $\eta$. From (3.8) and (3.12), the equation in $\eta$ becomes

$$
\left(\eta_{t}, \phi_{h}\right)+A\left(\mathscr{B}^{\alpha} \eta, \phi_{h}\right)+b\left(u, u, \phi_{h}\right)-b\left(u_{h}, u_{h}, \phi_{h}\right)=0,
$$

with $\eta(0)=0$.

Equivalently, the above equation can be recast as

$$
\eta_{t}+\mathscr{B}^{\alpha} \mathscr{L} \eta+B(u, u)-B\left(u_{h}, u_{h}\right)=0, \quad \eta(0)=0 .
$$

By Duhamel's principle(cf. [48]) and Lemma 3.1 with $p=1, q=0$, one can derive that

$$
\begin{aligned}
\|\eta\| & =\left\|\int_{0}^{t} E_{h}(t-s)\left(B(u, u)-B\left(u_{h}, u_{h}\right)\right) d s\right\| \\
& \leq \int_{0}^{t}\left\|\mathscr{F}^{1 / 2} E_{h}(t-s) \mathscr{F}^{-1 / 2}\left(B(u, u)-B\left(u_{h}, u_{h}\right)\right)\right\| d s \\
& \leq \int_{0}^{t}(t-s)^{-\alpha / 2}\left\|\mathscr{F}^{-1 / 2}\left(B(u, u)-B\left(u_{h}, u_{h}\right)\right)\right\| d s,
\end{aligned}
$$

where $\mathscr{F}$ denotes the discrete Laplace operator. Thus it is enough to estimate $\| \mathscr{F}^{-1 / 2}(B(u, u)-$ $\left.B\left(u_{h}, u_{h}\right)\right) \|$. We proceed by the standard duality arguments, using the splitting

$$
B(u, u)-B\left(u_{h}, u_{h}\right)=B(u, e)+B\left(e, u_{h}\right) .
$$

By the triangle inequality, it yields

$$
\left\|\mathscr{F}^{-1 / 2}\left(B(u, u)-B\left(u_{h}, u_{h}\right)\right)\right\| \leq\|B(u, e)\|_{-1}+\left\|B\left(e, u_{h}\right)\right\|_{-1},
$$

so that the proof is reduced to estimate each of the above negative norms on the right-hand side. Using the skew-symmetry property (3.6) and noticing $\operatorname{div} u=0$, one obtains for the first term:

$$
\begin{aligned}
\|B(u, e)\|_{-1} & =\sup _{\|\phi\|=1}\left|-((u \cdot \nabla) \phi, e)-\frac{1}{2}((\nabla \cdot u) \phi, e)\right| \\
& \leq \sup _{\|\phi\|=1}\left(\|e\|\|u\|_{\infty}\|\phi\|_{1}+\|e\|\|\nabla \cdot u\|_{L^{4}}\|\phi\|_{L^{4}}\right) \leq C\|e\| .
\end{aligned}
$$

Regarding the other term in (3.17), we derive

$$
\begin{aligned}
\left\|B\left(e, u_{h}\right)\right\|_{-1} & =\sup _{\|\phi\|=1}\left|\frac{1}{2}\left((e \cdot \nabla) u_{h}, \phi\right)-\frac{1}{2}\left((e \cdot \nabla) \phi, u_{h}\right)\right| \\
& \leq \sup _{\|\phi\|=1}\left(\|e\|\left\|\nabla u_{h}\right\|_{L^{4}}\|\phi\|_{L^{4}}+\|e\|\|\phi\|_{1}\left\|u_{h}\right\|_{\infty}\right) \leq C\|e\|,
\end{aligned}
$$


where, in the last inequality, we have used the Sobolev's imbeddings $\|\phi\|_{L^{4}} \leq C\|\phi\|_{1}$. It is obvious that there holds

$$
\left\|\mathscr{F}^{-1 / 2}\left(B(u, u)-B\left(u_{h}, u_{h}\right)\right)\right\| \leq C\|e\| .
$$

Substituting (3.18) in (3.16), we can show that

$$
\|\eta\| \leq C \int_{0}^{t}(t-s)^{-\alpha / 2}\left\|u(s)-u_{h}(s)\right\| d s .
$$

By Gronwall's lemma, a use of the triangle inequality with Lemma 3.3 completes the rest of the proof.

\section{Full Discretization}

In this section, firstly following the idea of Zhuang et al. [53] that discretize the RiemannLiouville fractional derivative $\mathscr{B}^{\alpha}$ in time, then we adopt the finite difference method in time and obtain the stability and convergence properties related to the time discretization. By collecting the convergence results for the space discretization and for the time discretization, the error estimate for fully discrete scheme of (1.1) has been obtained.

We suppose $t_{n}=n \triangle t, n=0,1, \cdots, N$, in which $k:=\Delta t=\frac{T}{N}$ denotes the step of time. In the subsequence, we will give several lemmas which play an important role in the time discretization.

Firstly, we will give the numerical scheme to discretize the temporal fractional integral.

Lemma 4.1 ([53]). If $u(t) \in C^{1}[0, T], 0<\alpha<1$, then

$$
\mathscr{I}^{\alpha} u\left(t_{n}\right)=\frac{k^{\alpha}}{\Gamma(\alpha+1)} \sum_{j=0}^{n-1} b_{j}^{(\alpha)} u\left(t_{n-j}\right)+r^{(1)},
$$

where $\left|r^{(1)}\right| \leq c t_{n}^{\alpha} k, b_{j}^{(\alpha)}=(j+1)^{\alpha}-j^{\alpha}, j=0,1, \cdots, N$.

The coefficients $b_{n}^{(\alpha)}$ possess the following properties.

Lemma $4.2([50])$. In (4.1), the coefficients $b_{n}^{(\alpha)}(n=0,1, \cdots, N)$ satisfy the following properties:

(i) $b_{0}^{(\alpha)}=1, b_{n}^{(\alpha)}>0, n=0,1, \cdots, N$,

(ii) $b_{n}^{(\alpha)}>b_{n+1}^{(\alpha)}, n=0,1, \cdots, N$,

(iii) there exists a positive constant $c>0$ such that $k \leq c b_{n}^{(\alpha)} k^{\alpha}$.

Based on Lemma 4.1, in the sequel we will give the approximation of the Riemann-Liouville fractional derivative $\mathscr{B}^{\alpha}$ about time.

Lemma 4.3 ([53]). If $u(t) \in C^{2}[0, T]$, then

$$
\mathscr{B}^{\alpha} u\left(t_{n}\right)=\frac{k^{\alpha-1}}{\Gamma(\alpha+1)}\left[u\left(t_{n}\right)+\sum_{j=1}^{n-1}\left(b_{j}^{(\alpha)}-b_{j-1}^{(\alpha)}\right) u\left(t_{n-j}\right)\right]+R_{1}^{(n)},
$$

where $\left|R_{1}^{(n)}\right| \leq c b_{n-1}^{(\alpha)} k^{\alpha}$. 
Let

$$
\mathscr{L}^{\alpha} u_{h}\left(t_{n}\right):=\frac{k^{\alpha-1}}{\Gamma(\alpha+1)}\left[u_{h}\left(t_{n}\right)+\sum_{j=1}^{n-1}\left(b_{j}^{(\alpha)}-b_{j-1}^{(\alpha)}\right) u_{h}\left(t_{n-j}\right)\right],
$$

then $\mathscr{B}^{\alpha} u_{h}\left(t_{n}\right)=\mathscr{L}^{\alpha} u_{h}\left(t_{n}\right)+R_{1}^{(n)}$.

In order to simplify the notations and without loss of generality, we consider the case $f(x, t)=0$ in the scheme construction and its numerical analysis.

With the help of Lemma 4.3 and using $\mathscr{L}^{\alpha} u_{h}\left(t_{n}\right)$ as an approximation of $\mathscr{B}^{\alpha} u_{h}\left(t_{n}\right)$ in the equation (3.5), then we will get the fully discrete scheme of (1.1): find $\left(u_{h}^{n}, p_{h}^{n}\right) \in\left(X_{h}, M_{h}\right)$ such that for all $\left(\phi_{h}, q_{h}\right) \in\left(X_{h}, M_{h}\right)$,

$$
\begin{gathered}
\left(u_{h}^{n}, \phi_{h}\right)=\left(u_{h}^{n-1}, \phi_{h}\right)-d_{1}\left(\left(\nabla u_{h}^{n}, \nabla \phi_{h}\right)+\sum_{j=1}^{n-1}\left(b_{j}^{(\alpha)}-b_{j-1}^{(\alpha)}\right)\left(\nabla u_{h}^{n-j}, \nabla \phi_{h}\right)\right) \\
-k b\left(u_{h}^{n}, u_{h}^{n}, \phi_{h}\right)+k d\left(\phi_{h}, p_{h}^{n}\right)-k d\left(u_{h}^{n}, q_{h}\right),
\end{gathered}
$$

where $n=0,1, \cdots, N, d_{1}=\frac{\nu k^{\alpha}}{\Gamma(\alpha+1)}$.

The stability analysis for the fully discrete scheme of (1.1) is given in the following lemma.

Lemma 4.4. Let

$$
E_{n}=\left\|u_{h}^{n}\right\|^{2}+d_{1} \sum_{j=0}^{n-1} b_{j}^{(\alpha)}\left\|\nabla u_{h}^{n-j}\right\|^{2} .
$$

Then the fully discrete problem (4.3) is unconditionally stable. Besides, it holds

$$
E_{n} \leq E_{n-1} \leq \cdots \leq\left\|u_{h}^{0}\right\|^{2}
$$

where $n=0,1, \cdots, N$.

Proof. Setting $\phi_{h}=u_{h}^{n} \in X_{h}$ and $q_{h}=p_{h}^{n} \in M_{h}$ in (4.3), and making use of the property (3.6) of $b\left(u_{h}, v_{h}, w_{h}\right)$, we obtain

$$
\left(u_{h}^{n}, u_{h}^{n}\right)=\left(u_{h}^{n-1}, u_{h}^{n}\right)-d_{1}\left(\left(\nabla u_{h}^{n}, \nabla u_{h}^{n}\right)+\sum_{j=1}^{n-1}\left(b_{j}^{(\alpha)}-b_{j-1}^{(\alpha)}\right)\left(\nabla u_{h}^{n-j}, \nabla u_{h}^{n}\right)\right) .
$$

Using the inequality $(u, v) \leq \frac{1}{2}\left(\|u\|^{2}+\|v\|^{2}\right)$ and noting that $b_{j-1}^{(\alpha)}>b_{j}^{(\alpha)}$, we derive

$$
\left\|u_{h}^{n}\right\|^{2} \leq \frac{1}{2}\left(\left\|u_{h}^{n-1}\right\|^{2}+\left\|u_{h}^{n}\right\|^{2}\right)-d_{1}\left\|\nabla u_{h}^{n}\right\|^{2}+\frac{d_{1}}{2} \sum_{j=1}^{n-1}\left(b_{j-1}^{(\alpha)}-b_{j}^{(\alpha)}\right)\left(\left\|\nabla u_{h}^{n-j}\right\|^{2}+\left\|\nabla u_{h}^{n}\right\|^{2}\right) .
$$

Further, with Lemma 4.2, the above expression can be organized into

$$
\begin{aligned}
\frac{1}{2}\left\|u_{h}^{n}\right\|^{2} & \leq \frac{1}{2}\left\|u_{h}^{n-1}\right\|^{2}-d_{1}\left\|\nabla u_{h}^{n}\right\|^{2}+\frac{d_{1}}{2} \sum_{j=1}^{n-1}\left(b_{j-1}^{(\alpha)}-b_{j}^{(\alpha)}\right)\left\|\nabla u_{h}^{n-j}\right\|^{2}+\frac{d_{1}\left(1-b_{n-1}^{(\alpha)}\right)}{2}\left\|\nabla u_{h}^{n}\right\|^{2} \\
& \leq \frac{1}{2}\left\|u_{h}^{n-1}\right\|^{2}-\frac{d_{1}\left(1+b_{n-1}^{(\alpha)}\right)}{2}\left\|\nabla u_{h}^{n}\right\|^{2}+\frac{d_{1}}{2} \sum_{j=1}^{n-1}\left(b_{j-1}^{(\alpha)}-b_{j}^{(\alpha)}\right)\left\|\nabla u_{h}^{n-j}\right\|^{2} \\
& \leq \frac{1}{2}\left\|u_{h}^{n-1}\right\|^{2}-\frac{d_{1}}{2}\left\|\nabla u_{h}^{n}\right\|^{2}+\frac{d_{1}}{2} \sum_{j=1}^{n-1} b_{j-1}^{(\alpha)}\left\|\nabla u_{h}^{n-j}\right\|^{2}-\frac{d_{1}}{2} \sum_{j=1}^{n-1} b_{j}^{(\alpha)}\left\|\nabla u_{h}^{n-j}\right\|^{2}
\end{aligned}
$$


which can be simplified as

$$
\left\|u_{h}^{n}\right\|^{2}+d_{1} \sum_{j=0}^{n-1} b_{j}^{(\alpha)}\left\|\nabla u_{h}^{n-j}\right\|^{2} \leq\left\|u_{h}^{n-1}\right\|^{2}+d_{1} \sum_{j=0}^{n-2} b_{j}^{(\alpha)}\left\|\nabla u_{h}^{n-1-j}\right\|^{2} .
$$

In other words, we obtained the desired asseat (4.4).

The error analysis for the solution of the semi-discrete problem about time is discussed in the following theorem.

Theorem 4.1 (Error estimate for time discretization). Let $u_{h}\left(t_{n}\right)$ be the solution of (3.5), $\left\{u_{h}^{n}\right\}_{n=1}^{N}$ be the time-discrete solution of (4.3). Under the assumptions of a small initial data and a small time step size, then we have the following error estimate

$$
\left\|u_{h}\left(t_{n}\right)-u_{h}^{n}\right\| \leq c k .
$$

Proof. Let $e^{n}=u_{h}\left(t_{n}\right)-u_{h}^{n}, \tilde{e}^{n}=p_{h}\left(t_{n}\right)-p_{h}^{n}$. Subtracting (4.3) from (3.5), the error equation can be written as

$$
\begin{gathered}
\left(e^{n}, \phi_{h}\right)=\left(e^{n-1}, \phi_{h}\right)-d_{1}\left(\left(\nabla e^{n}, \nabla \phi_{h}\right)+\sum_{j=1}^{n-1}\left(b_{j}^{(\alpha)}-b_{j-1}^{(\alpha)}\right)\left(\nabla e^{n-j}, \nabla \phi_{h}\right)\right) \\
-k b\left(e^{n}, u_{h}\left(t_{n}\right), e^{n}\right)+k d\left(\phi_{h}, \tilde{e}^{n}\right)-k d\left(e^{n}, q_{h}\right)+k\left(R_{1}^{n}, \phi_{h}\right) .
\end{gathered}
$$

Taking $\phi_{h}=e^{n}, q_{h}=\tilde{e}^{n}$ in (4.5), there holds

$$
\begin{gathered}
\left(e^{n}, e^{n}\right)=\left(e^{n-1}, e^{n}\right)-d_{1}\left(\left(\nabla e^{n}, \nabla e^{n}\right)+\sum_{j=1}^{n-1}\left(b_{j}^{(\alpha)}-b_{j-1}^{(\alpha)}\right)\left(\nabla e^{n-j}, \nabla e^{n}\right)\right) \\
-k b\left(e^{n}, u_{h}\left(t_{n}\right), e^{n}\right)+k\left(R_{1}^{n}, e^{n}\right) .
\end{gathered}
$$

Making use of the inequality $(u, v) \leq \frac{1}{2}\left(\|u\|^{2}+\|v\|^{2}\right)$ and noting that $b_{j-1}^{(\alpha)}>b_{j}^{(\alpha)}$, we observe that

$$
\begin{aligned}
\left\|e^{n}\right\|^{2} \leq \frac{1}{2}\left(\left\|e^{n-1}\right\|^{2}+\left\|e^{n}\right\|^{2}\right)-d_{1}\left\|\nabla e^{n}\right\|^{2} & \\
& +\frac{d_{1}}{2} \sum_{j=1}^{n-1}\left(b_{j-1}^{(\alpha)}-b_{j}^{(\alpha)}\right)\left(\left\|\nabla e^{n-j}\right\|^{2}+\left\|\nabla e^{n}\right\|^{2}\right)-k b\left(e^{n}, u_{h}\left(t_{n}\right), e^{n}\right)+k\left(R_{1}^{n}, e^{n}\right),
\end{aligned}
$$

which can be simplified as

$$
\begin{aligned}
\left\|e^{n}\right\|^{2} \leq\left\|e^{n-1}\right\|^{2}-d_{1} \sum_{j=0}^{n-1} b_{j}^{(\alpha)}\left\|\nabla e^{n-j}\right\|^{2}-d_{1} b_{n-1}^{(\alpha)}\left\|\nabla e^{n}\right\|^{2} \\
+d_{1} \sum_{j=0}^{n-2} b_{j}^{(\alpha)}\left\|\nabla e^{n-1-j}\right\|^{2}-2 k b\left(e^{n}, u_{h}\left(t_{n}\right), e^{n}\right)+2 k\left(R_{1}^{n}, e^{n}\right) .
\end{aligned}
$$

Let $Y_{n}=\left\|e^{n}\right\|^{2}+d_{1} \sum_{j=0}^{n-1} b_{j}^{(\alpha)}\left\|\nabla e^{n-j}\right\|^{2}$, then the above expression can be read as

$$
Y_{n} \leq Y_{n-1}-d_{1} b_{n-1}^{(\alpha)}\left\|\nabla e^{n}\right\|^{2}-2 k b\left(e^{n}, u_{h}\left(t_{n}\right), e^{n}\right)+2 k\left(R_{1}^{n}, e^{n}\right) .
$$


Applying the property $(2.1)$ of $b(u, v, w)$, we have

$$
-2 k b\left(e^{n}, u_{h}, e^{n}\right) \leq 2 k M\left\|e^{n}\right\|_{1}^{2}\left\|u_{h}\right\|,
$$

note that the right hand side of the above inequality can be sufficiently small which can be derived from (3.11) only if it requires a small initial data and a small time step size. Making use of the Sobolev's imbeddings $\left\|e^{n}\right\| \leq \gamma\left\|\nabla e^{n}\right\|$, the inequality (4.6) can be written as

$$
Y_{n} \leq Y_{n-1}-\frac{d_{1} b_{n-1}^{(\alpha)}}{\gamma^{2}}\left\|e^{n}\right\|^{2}+2 k\left(R_{1}^{n}, e^{n}\right) .
$$

Note that

$$
(\alpha(x), \beta(x)) \leq \lambda\|\alpha(x)\|^{2}+\frac{1}{4 \lambda}\|\beta(x)\|^{2}, \quad(\lambda>0) .
$$

Hence,

$$
2 k\left(R_{1}^{n}, e^{n}\right) \leq \frac{d_{1} b_{n-1}^{(\alpha)}}{\gamma^{2}}\left\|e^{n}\right\|^{2}+\frac{\gamma^{2}}{4 d_{1} b_{n-1}^{(\alpha)}}\left\|2 k R_{1}^{n}\right\|^{2} .
$$

Substituting (4.8) into (4.7), it holds

$$
Y_{n} \leq Y_{n-1}+\frac{\gamma^{2} k^{2}}{d_{1} b_{n-1}^{(\alpha)}}\left\|R_{1}^{n}\right\|^{2} \leq Y_{n-1}+\frac{\gamma^{2}}{\nu} \Gamma(\alpha+1) b_{n-1}^{(\alpha)} k^{\alpha+2},
$$

the last inequality of which can be obtained from $d_{1}=\frac{\nu k^{\alpha}}{\Gamma(\alpha+1)}$ and $\left|R_{1}^{(n)}\right| \leq c b_{n-1}^{(\alpha)} k^{\alpha}$. Since $Y_{0}=e^{0}=0$, it derives that

$$
\begin{aligned}
Y_{n} & \leq \frac{\gamma^{2}}{\nu} \Gamma(\alpha+1) k^{\alpha+2} \sum_{j=1}^{n} b_{j-1}^{(\alpha)} \\
& =\frac{\gamma^{2}}{\nu} \Gamma(\alpha+1) k^{\alpha+2} \sum_{j=1}^{n}\left(j^{\alpha}-(j-1)^{\alpha}\right) \\
& \leq \frac{\gamma^{2}}{\nu} \Gamma(\alpha+1) T^{\alpha} k^{2} \leq c k^{2},
\end{aligned}
$$

where $c=\frac{\gamma^{2}}{\nu} \Gamma(\alpha+1) T^{\alpha}$. The proof is completed.

Next we will give the error estimate for the fully discrete scheme by collecting the convergence results for the space discretization and for the time discretization.

Theorem 4.2 (Error estimate for fully discrete scheme). Let $\left\{u\left(t_{n}\right)\right\}$ be the solution of (1.1) and let $\left\{u_{h}^{n}\right\}_{n=1}^{N}$ be the solution of the scheme (4.3) with $T=N \triangle t$. Under the assumptions of a small initial data and a small time step size, then there is $c>0$ such that

$$
\left\|u\left(t_{n}\right)-u_{h}^{n}\right\| \leq c\left(h^{2}+k\right)
$$

Proof. The proof follows from Theorems 3.1 and 4.1 by the triangle inequality. We are no longer to repeat here. 


\section{Numerical Experiment}

In order to demonstrate the effectiveness of our numerical methods, numerical examples are presented. The main purpose is to check the convergence behavior of the numerical solution with respect to the time step $\Delta t$ and the space step $h$ used in the calculation.

We consider the following fractional equation

$$
\begin{array}{ll}
u_{t}-\mathscr{B}^{\alpha} u_{x x}+u u_{x}=f(x, t), & x \in \Omega, t \in[0,1], \\
u(0, t)=u(1, t)=0, & t \in[0,1], \\
u(x, 0)=0, & x \in \Omega,
\end{array}
$$

where $\Omega=[0,1]$.

We compute the errors in $L^{2}$ discrete norm. And all the numerical results in the tables below are evaluated at $T=1$. The spatial and temporal meshes are taken uniform. The finite element method using piecewise-linear polynomials is used for the space and the scheme for time described in previous sections is used in the examples.

Example 5.1. The source term $f$ is chosen as

$$
f(x, t)=2 t \sin (\pi x)+\frac{2(\pi)^{2}}{\Gamma(2+\alpha)} t^{1+\alpha} \sin (\pi x)+\pi t^{4} \sin (\pi x) \cos (\pi x) .
$$

Then the exact solution is $u(x, t)=t^{2} \sin (\pi x)$.

For the $0<\alpha<1$ case, the theoretical convergence order is $O\left(h^{2}+k\right)$. To examine the rate of convergence for this method, in Table 1 , for a fixed time step $\Delta t=1 / 800$ and some different spatial meshes, we can see the orders of convergence for $u$ in $L^{2}$-norms are close to 2 which are accord with the spatial convergence order $O\left(h^{2}\right)$. In Table 2, for a fixed spatial step $h=1 / 1000$, it shows that the orders of convergence for $u$ in $L^{2}$-norms are close to 1 which are accord with the time convergence order $O(k)$. The numerical results are consistent with our theoretical results in Theorem 4.2.

Table 5.1: The errors and space convergence rates for $u$ with fixed time step $\Delta t=1 / 800$.

\begin{tabular}{|lllllll|}
\hline \multirow{2}{*}{$h$} & \multicolumn{2}{c}{$\alpha=0.3$} & \multicolumn{2}{c|}{$\alpha=0.6$} & \multicolumn{2}{c|}{$\alpha=0.9$} \\
\cline { 2 - 7 } & $\left\|u(T)-u_{h}^{N}\right\|$ & cv.rate & $\left\|u(T)-u_{h}^{N}\right\|$ & cv.rate & $\left\|u(T)-u_{h}^{N}\right\|$ & cv.rate \\
\hline $1 / 4$ & $3.5673 \mathrm{E}-2$ & - & $3.4926 \mathrm{E}-2$ & - & $3.4058 \mathrm{E}-2$ & - \\
$1 / 8$ & $8.8397 \mathrm{E}-3$ & 2.013 & $8.6821 \mathrm{E}-3$ & 2.008 & $8.4523 \mathrm{E}-3$ & 2.011 \\
$1 / 16$ & $2.1018 \mathrm{E}-3$ & 2.072 & $2.1059 \mathrm{E}-3$ & 2.044 & $2.0528 \mathrm{E}-3$ & 2.042 \\
$1 / 32$ & $4.2061 \mathrm{E}-4$ & 2.321 & $4.6254 \mathrm{E}-4$ & 2.187 & $4.5466 \mathrm{E}-4$ & 2.174 \\
\hline
\end{tabular}

Example 5.2. We choose the exact solution $u(x, t)=t^{1 / 2} \sin (\pi x)$, the smoothing property of which is less. Then the source term can be arrived at

$$
f(x, t)=\frac{1}{2} t^{-\frac{1}{2}} \sin (\pi x)+\frac{\Gamma(3 / 2) \pi^{2}}{\Gamma(1 / 2+\alpha)} t^{\alpha-1 / 2} \sin (\pi x)+\pi t \sin (\pi x) \cos (\pi x),
$$

where $f(x, t)$ is nonsmooth in time.

In Table 3, for a fixed time step $\Delta t=1 / 5000$ and some different spatial meshes, we can see the orders of convergence for $u$ in $L^{2}$-norms are close to 2 which are in agreement with the spatial 
Table 5.2: The errors and time convergence rates for $u$ with fixed space step $h=1 / 1000$.

\begin{tabular}{|lllllll|}
\hline \multirow{2}{*}{$\Delta t$} & \multicolumn{2}{c}{$\alpha=0.3$} & \multicolumn{2}{c|}{$\alpha=0.6$} & \multicolumn{2}{c|}{$\alpha=0.9$} \\
\cline { 2 - 7 } & $\left\|u(T)-u_{h}^{N}\right\|$ & cv.rate & $\left\|u(T)-u_{h}^{N}\right\|$ & cv.rate & $\left\|u(T)-u_{h}^{N}\right\|$ & cv.rate \\
\hline $1 / 8$ & $3.7512 \mathrm{E}-2$ & - & $1.7726 \mathrm{E}-2$ & - & $1.0798 \mathrm{E}-2$ & - \\
$1 / 16$ & $1.6424 \mathrm{E}-2$ & 1.192 & $7.5884 \mathrm{E}-3$ & 1.224 & $5.0085 \mathrm{E}-3$ & 1.108 \\
$1 / 32$ & $7.1783 \mathrm{E}-3$ & 1.194 & $3.3325 \mathrm{E}-3$ & 1.187 & $2.4005 \mathrm{E}-3$ & 1.061 \\
$1 / 64$ & $3.1469 \mathrm{E}-3$ & 1.189 & $1.5045 \mathrm{E}-3$ & 1.147 & $1.1738 \mathrm{E}-3$ & 1.032 \\
\hline
\end{tabular}

Table 5.3: The errors and space convergence rates.

\begin{tabular}{|lllll|}
\hline$\alpha$ & $\Delta t$ & $\mathrm{~h}$ & $\left\|u(T)-u_{h}^{N}\right\|$ & cv.rate \\
\hline 0.1 & $1 / 5000$ & $1 / 2$ & $1.4513 \mathrm{E}-1$ & - \\
& $1 / 5000$ & $1 / 4$ & $3.9126 \mathrm{E}-2$ & 1.891 \\
& $1 / 5000$ & $1 / 8$ & $1.1972 \mathrm{E}-2$ & 1.708 \\
0.3 & $1 / 5000$ & $1 / 2$ & $1.4382 \mathrm{E}-1$ & - \\
& $1 / 5000$ & $1 / 4$ & $3.7275 \mathrm{E}-2$ & 1.948 \\
& $1 / 5000$ & $1 / 8$ & $9.9406 \mathrm{E}-3$ & 1.907 \\
0.9 & $1 / 5000$ & $1 / 2$ & $1.4572 \mathrm{E}-1$ & - \\
& $1 / 5000$ & $1 / 4$ & $3.7357 \mathrm{E}-2$ & 1.967 \\
& $1 / 5000$ & $1 / 8$ & $9.4572 \mathrm{E}-3$ & 1.982 \\
\hline
\end{tabular}

Table 5.4: The errors and time convergence rates.

\begin{tabular}{|lllll|}
\hline$\alpha$ & $h$ & $\Delta t$ & $\left\|u(T)-u_{h}^{N}\right\|$ & cv.rate \\
\hline 0.3 & $1 / 1000$ & $1 / 10$ & $5.3429 \mathrm{E}-2$ & - \\
& $1 / 1000$ & $1 / 20$ & $3.3131 \mathrm{E}-2$ & 0.703 \\
& $1 / 1000$ & $1 / 40$ & $2.0364 \mathrm{E}-2$ & 0.702 \\
0.6 & $1 / 1000$ & $1 / 10$ & $1.8203 \mathrm{E}-2$ & - \\
& $1 / 1000$ & $1 / 20$ & $1.0321 \mathrm{E}-2$ & 0.819 \\
& $1 / 1000$ & $1 / 40$ & $6.1121 \mathrm{E}-3$ & 0.756 \\
0.9 & $1 / 1000$ & $1 / 10$ & $4.2612 \mathrm{E}-3$ & - \\
& $1 / 1000$ & $1 / 20$ & $2.3878 \mathrm{E}-3$ & 0.836 \\
& $1 / 1000$ & $1 / 40$ & $1.4430 \mathrm{E}-3$ & 0.727 \\
\hline
\end{tabular}

convergence order $O\left(h^{2}\right)$. Table 4 exhibits the numerical errors in temporal direction with different $\alpha$ for a fixed sufficiently small spatial step. Because the source term $f$ is nonsmooth in time, the convergence for small fractional order $\alpha$ suffers some loss.

All in all, based on the above numerical examples, one can find that our numerical method is effective.

Acknowledgments. The authors want to thank Prof. Yang Liu, Inner Mongolia University, China, for his kindness and help with the numerical example. The authors would like to express their sincere gratitude to the anonymous reviewers for their careful reading of the manuscript, as well as their comments that lead to a considerable improvement of the original manuscript. The second author was supported by the Beijing Municipal Commission of Education Science and Technology Program Project KM201810017009, Beijing Municipal Natural Science Foundation under Grant 4202028. 


\section{References}

[1] G.A. Baker, Galerkin approximations for the Navier-Stokes equations, Manuscript, Harvard University, Cambridge, MA, 1976.

[2] C. Bernardi and G. Raugel, A conforming finite element method for the time-dependent NavierStokes equations, Siam J. Numer. Anal., 22:3 (1985), 455-473.

[3] D.L. Brown, R. Cortez and M. L. Minion, Accurate projection methods for the incompressible Navier-Stokes equations, J. Comput. Phys., 168:2 (2001), 464-499.

[4] E. Burman, Pressure projection stabilizations for Galerkin approximations of Stokes and Darcys problem, Numer. Meth. Part. D. E., 24 (2008), 127-143.

[5] W. Bu, Y. Tang and J. Yang, Galerkin finite element method for two-dimensional Riesz space fractional diffusion equations, J. Comput. Phys., 276 (2014), 26-38.

[6] W.H. Deng, Finite element method for the space and time fractional Fokker-Planck equation, SIAM J. Numer. Anal., 47:1 (2008), 204-226.

[7] W.H. Deng and J.S. Hesthaven, Local discontinuous Galerkin methods for fractional ordinary differential equations, BIT, 55 (2015), 967-985.

[8] C. Févrière, J. Laminie, P. Poullet and P. Poullet, On the penalty-projection method for the Navier-Stokes equations with the MAC mesh, J. Comput. Appl. Math., 226 (2009), 228-245.

[9] J.D. Frutos, B. Garca-Archilla and J. Novo, Optimal error bounds for two-grid schemes applied to the Navier-Stokes equations, Appl. Math. Comput., 218:13 (2012), 7034-7051.

[10] V. Girault and P.A. Raviart, Finite Element Methods for Navier-Stokes Equations: Theory and Algorithms, Springer-Verlag, Berlin Heidelberg, 1986.

[11] D. Goswami and P.D. Damázio, A two-level finite element method for time-dependent incompressible Navier-Stokes equations with non-smooth initial data, arXiv:1211.3342 [math.NA].

[12] B.Y. Guo and Y.J. Jiao, Spectral method for Navier-Stokes equations with slip boundary conditions, J. Sci. Comput. 58 (2014), 249-274.

[13] J.G. Heywood and R. Rannacher, Finite element approximation of the nonstationary NavierStokes problem. I. Regularity of solutions and second-order spatial discretization, SIAM J. Numer. Anal., 19 (1982), 275-311.

[14] Y.N. He and W.W. Sun, Stability and convergence of the Crank-Nicolson/Adams-Bashforth scheme for the time-dependent Navier-Stokes equations, SIAM J. Numer. Anal., 45:2 (2007), $837-869$.

[15] Y.N. He and J. Li, Convergence of three iterative methods based on the finite element discretization for the stationary Navier-Stokes equations, Comput. Method. Appl. M., 198 (2009), 1351-1359.

[16] Y.N. He, Two-level method based on finite element and Crank-Nicolson extrapolation for the time-dependent Navier-Stokes equations, SIAM J. Numer. Anal., 41 (2004), 1263-1285.

[17] P. Huang, X. Feng and D. Liu, A stabilized finite element method for the time-dependent Stokes equations based on Crank-Nicolson Scheme, Appl. Math. Model., 37:4 (2013), 1910-1919.

[18] Y. He, P. Huang and X. Feng, $H^{2}$-stability of the first order fully discrete schemes for the timedependent Navier-Stokes equations, J. Sci. Comput., 62:1 (2015), 230-264.

[19] H. Johnston and J.G. Liu, Accurate, stable and efficient Navier-Stokes solvers based on explicit treatment of the pressure term, J. Comput. Phys., 199:1 (2004), 221-259.

[20] Y. Jiang and J. Ma, High-order finite element methods for time-fractional partial differential equations, J. Comput. Appl. Math., 235:11 (2011), 3285-3290.

[21] B. Jin, R. Lazarov and Z. Zhou, Error estimates for a semidiscrete finite element method for fractional order parabolic equations, SIAM J. Numer. Anal., 51:1 (2012), 445-466. 
[22] B. Jin, R. Lazarov, J. Pasciak and Z. Zhou, Error analysis of semidiscrete finite element methods for inhomogeneous time-fractional diffusion, IMA J. Numer. Anal., 2014.

[23] B. Jin, R. Lazarov and Y. Liu, The Galerkin finite element method for a multi-term time-fractional diffusion equation, J. Comput. Phys., 281 (2015), 825-843.

[24] B. Jin, R. Lazarov and Z. Zhou, An analysis of the L1 scheme for the subdiffusion equation with nonsmooth data, IMA J. Numer. Anal., 2015.

[25] J. Kim and P. Moin, Application of a fractional-step method to incompressible Navier-Stokes equations, J. Comput. Phys., 59:2 (1985), 308-323.

[26] A.A. Kilbas, H.M. Srivastava and J.J. Trujillo, Theory and Applications of Fractional Differential Equations, Elsevier, Amsterdam, 2006.

[27] Li X, Yang X, Zhang Y., Error Estimates of mixed finite element methods for time-fractional Navier-Stokes equations, J. Sci. Comput., 70:2 (2017), 500-515.

[28] S. Karaa, K. Mustapha and A.K. Pani, Finite volume element method for two-dimensional fractional subdiffusion problems, IMA J. Numer. Anal., (2015), 1-17.

[29] Y. Lin and L. Wahlbin, Ritz-Volterra projections to finite-element spaces and applications to integrodifferential and related equations, SIAM J. Numer. Anal., 28:4 (1991), 1047-1070.

[30] F. Liu, V. Anh, I. Turner and P. Zhuang, Time fractional advection dispersion equation, J. Appl. Math. Comput., 13 (2003), 233-245.

[31] Y. Lin and C. Xu, Finite difference/spectral approximations for the time-fractional diffusion equation, J. Comput. Phys., 225:2 (2007), 1533-1552.

[32] Q. Liu and Y. Hou, A two-level finite element method for the Navier-Stokes equations based on a new projection, Appl. Math. Model., 34:2 (2010), 383-399.

[33] C.P. Li, Z.G. Zhao and Y.Q. Chen, Numerical approximation of nonlinear fractional differential equations with subdiffusion and superdiffusion, Comput. Math. Appl., 62.3 (2011), 855-875.

[34] Z.D. Luo, A new finite volume element formulation for the non-stationary Navier-Stokes equations, Adv. Appl. Math. Mech., 6 (2014), 615-636.

[35] Y. Liu, Y. Du, H. Li and J. Li, A two-grid mixed finite element method for a nonlinear fourth-order reaction-diffusion problem with time-fractional derivative, Comput. Math. Appl., 70:10 (2015), 2474-2492.

[36] Y. Liu, Y. Du, H. Li, S. He and W. Gao, Finite difference/finite element method for a nonlinear time-fractional fourth-order reaction-diffusion problem, Comput. Math. Appl., 70:4 (2015), 573591.

[37] C.H. Min and F. Gibou, A second order accurate projection method for the incompressible NavierStokes equations on non-graded adaptive grids, J. Comput. Phys., 219 (2006), 912-929.

[38] R.H. Nochetto and J.H. Pyo, The Gauge-Uzawa finite element method. Part I: The Navier-Stokes equations, SIAM J. Numer. Anal., 43 (2005), 1043-1068.

[39] H. Okamoto, On the semi-discrete finite element approximation for the nonstationary NavierStokes equation, Journal of the Faculty of Science the University of Tokyo. sect A Math., 29:3 (1982), 613-651.

[40] A.K. Pani and J.Y. Yuan, Semidiscrete finite element Galerkin approximations to the equations of motion arising in the Oldroyd model, IMA J. Numer. Anal., 25:4 (2005), 750-782.

[41] R. Rannacher, Numerical analysis of the Navier-Stokes equations, Appl. Math., 38 (1993), 361380.

[42] T. Chacón Rebollo, M. Gómez Mármol and M. Restelli, Numerical analysis of penalty stabilized finite element discretizations of evolution Navier-Stokes equations, J. Sci. Comput., 63 (2015), 885-912.

[43] J. Shen, On error estimates of projection methods for the Navier-Stokes equations: second order schemes, Math. Comput., 65 (1996), 1039-1065.

[44] L. Shan and Y. Hou, A fully discrete stabilized finite element method for the time-dependent Navier-Stokes equations, Appl. Math. Comput., 215:1 (2009), 85-99. 
[45] S.S. Siddiqi and S. Arshed, Numerical solution of time-fractional fourth-order partial differential equations, Int. J. Comput. Math., 5 (2014), 1-23.

[46] R. Temam, Navier-Stokes Equations, Theory and Numerical Analysis, North-Holland, Amsterdam, 1984.

[47] F. Tone, Error analysis for a second order scheme for the Navier-Stokes equations, Appl. Numer. Math., 50:1 (2004), 93-119.

[48] V. Thomée, Galerkin Finite Element Methods for Parabolic Problems, Spriger Series in Computational Mathematics vol. 25, Springer-Verlag, Berlin Heidelberg, 1997.

[49] MF. Wheeler, A Priori $L_{2}$ Error Estimates for Galerkin Approximations to Parabolic Partial Differential Equations, SIAM J. Numer. Anal., 10:10 (1973), 723-759.

[50] P. Zhuang, F. Liu, V. Anh and I. Turner, New solution and analytical techniques of the implicit numerical method for the anomalous subdiffusion equation, SIAM J. Numer. Anal., 46 (2008), 1079-1095.

[51] F.H. Zeng, C.P. Li, F.W. Liu and I. Turner, Numerical algorithms for time-fractional subdiffusion equation with second-order accuracy, SIAM J. Sci. Comput., 37 (2015), 55-78.

[52] X.d. Zhang, J. Liu, L. Wei and C. Ma, Finite element method for GrwnwaldCLetnikov timefractional partial differential equation, Appl. Anal., 92 (2013), 2103-2114.

[53] P. Zhuang, F. Liu, I. Turner and V. Anh, Galerkin finite element method and error analysis for the fractional cable equation, Numer. Algorithms, (2015), 1-20.

[54] Lijing Zhao, Weihua Deng and Jan S Hesthaven, Spectral Methods for Tempered Fractional Differential Equations, 2016.

[55] G.A. Zou, G.Y. Lv and J.L. Wu, Stochastic Navier-Stokes equations with Caputo derivative driven by fractional noises, J. Math. Anal. Appl., 461:1 (2017), 595-609.

[56] G.A. Zou, Y.Zhou, Ahmad B, et al., Finite difference/element method for time-fractional NavierStokes equations, 2018.

[57] M. Gunzburger, B. Li and J. Wang, Sharp convergence rates of time discretization for stochastic time-fractional PDEs subject to additive space-time white noise, Math. Comput., (2017), DOI: $10.1090 / \mathrm{mcom} / 3397$. 